\title{
A Novel Method for Improving the Capacity in 5G Mobile Networks Combining NOMA and OMA
}

Marcano, Andrea S.; Christiansen, Henrik Lehrmann

Published in:

Vehicular Technology Conference (VTC Spring), 2017 IEEE 85th

Link to article, DOI:

10.1109/VTCSpring.2017.8108677

Publication date:

2017

Document Version

Peer reviewed version

Link back to DTU Orbit

Citation $(A P A)$ :

Marcano, A. S., \& Christiansen, H. L. (2017). A Novel Method for Improving the Capacity in 5G Mobile Networks Combining NOMA and OMA. In Vehicular Technology Conference (VTC Spring), 2017 IEEE 85th IEEE. https://doi.org/10.1109/VTCSpring.2017.8108677

\section{General rights}

Copyright and moral rights for the publications made accessible in the public portal are retained by the authors and/or other copyright owners and it is a condition of accessing publications that users recognise and abide by the legal requirements associated with these rights.

- Users may download and print one copy of any publication from the public portal for the purpose of private study or research.

- You may not further distribute the material or use it for any profit-making activity or commercial gain

- You may freely distribute the URL identifying the publication in the public portal 


\title{
A novel method for improving the capacity in $5 \mathrm{G}$ mobile networks combining NOMA and OMA
}

\author{
Andrea S. Marcano and Henrik L. Christiansen \\ DTU Fotonik, Technical University of Denmark \\ anmarc@fotonik.dtu.dk; hlch@fotonik.dtu.dk
}

\begin{abstract}
Non-Orthogonal Multiple Access (NOMA) has been suggested as one of the technologies to be implemented in the fifth generation - 5G - mobile networks. NOMA helps increasing the capacity by offering a more effective use of the available resources, but it also intentionally introduces interference in the transmitted signal. This means that higher signal-to-interference-plus-noise ratio (SINR) values are required to decode the received signal, in comparison to orthogonal multiple access (OMA). Since it is unrealistic to consider a system working only with NOMA because not all the users (UEs) will meet the requirements to use this multiple access (MA) method, a hybrid MA system combining NOMA with OMA is expected. In this paper we present the performance evaluation of a hybrid MA system combined with a novel pairing algorithm based on modulation and coding scheme (MCS) adjustment and extra transmission power ( $T x$ power) allocation. The purpose of such pairing approach is to help the NOMA UEs reach the needed SINR while improving the overall system capacity. Moreover, we use mmWave for the signal propagation to further increase the system capacity. The results show that such a system with an extra Tx power between $14 \%$ and $19 \%$ of the total $T x$ power for OMA, can offer the best tradeoff with a system capacity gain up to 1.78 -fold for a total of $6.7 \mathrm{Gbps}$, an average 3.31-fold increase for the UEs bit rate, and a block error rate (BLER) below $10 \%$.
\end{abstract}

Keywords-NOMA, hybrid MA, capacity, power allocation, BLER, SINR, MCS, $5 G$

\section{INTRODUCTION}

Among the key capabilities expected for the fifth generation of mobile networks - 5G networks -, higher system capacity is one of the most important and challenging. 5G networks must be able to cope with the continuously increasing data demand that will be driven mainly by the Internet of Things (IoT) and video streaming applications. The maximum achievable bit rate per user/device in $5 \mathrm{G}$ networks should reach values in the order of Gbits/s under ideal conditions, and ubiquitous connection in the order of Mbits/s or Gbits/s should be guaranteed across the coverage area of the network[1]. Nonorthogonal multiple access (NOMA) is one of the radio access technologies proposed so far as a possible option to satisfy the future capacity demands[2].

NOMA, unlike the orthogonal multiple access (OMA) methods, exploits the power domain by multiplexing the users (UEs) and intentionally introducing interference by sharing the same resources without spatial separation. This can be done by implementing superposition techniques [4]. NOMA have been considered as part of possible implementations for $5 \mathrm{G}$ networks, the works in [2]-[4] are some examples. Our work in [5] presents a performance analysis for a 5G NOMA system combined with mmWave frequencies; the results showed that although up to $70 \%$ of channel capacity gain can be achieved by implementing NOMA instead of OMA, a penalty of about $12 \mathrm{~dB}$ in the minimum required signal-to-interference-plusnoise ratio (SINR) is needed in order to maintain a block error rate (BLER) below 10\%. Furthermore, in [5] it is concluded that a successful signal decoding with NOMA is possible only when a QPSK modulation is assigned to one of the power multiplexed UE. This means that not all the UEs within the cell can be paired. Hence, it is sub-optimal to consider NOMA as the only multiple access (MA) method; on the contrary, a hybrid system combining NOMA with OMA is expected, along with possible modulation and coding scheme (MCS) adjustment to make up for the extra SINR, when needed.

The implementation of such hybrid system has also been suggested by other authors. In [4], cooperative NOMA is suggested as a technique to improve the signal decoding, but due to the complexity of a NOMA only system, a hybrid MA approach is suggested. In [6], the performance of a system switching between three types of non-orthogonal transmissions and OMA is evaluated.

Moreover, the work in [7] shows how the combinations of NOMA and OMA offers the largest rate regions in a two-user interference channel, following the Han-Kobayashi scheme [8], which it its basic form implies NOMA. The works in [9] and [10] focus on the improvement in spectral efficiency of multicarrier (MC) NOMA. In [9] an optimal power and subcarrier allocation algorithm for full-duplex MC-NOMA, designed to maximized the weighted sum throughput in such, is proposed. In [10] also an optimal power allocation is proposed for MC-NOMA based on quality of service (QoS) constrains under statistical channel state information (CSI) at the transmitter. Both [9] and [10] compare the performance of the proposed methods with OMA systems, showing that a NOMA implementation outperforms OMA in terms of spectral efficiency.

Up to date, no previous studies have evaluated the performance of a hybrid MA system focusing on techniques that help increasing the total system capacity while maintaining desirable BLER values. The purpose of this paper is to evaluate the UEs bit rate gain, system capacity gain, power consumption, and BLER, of a system that implements hybrid MA scheduling. We combine this system with a pairing method for NOMA based on MCS adjustment and extra transmission power (Tx power) allocation in order to reach the needed SINR values and maintain a BLER below $10 \%$. Moreover, we combine this system with mmWave wireless transmission to further increase the overall system capacity. 


\section{NOMA FUNDAMENTALS}

\section{A. NOMA Overview}

MA techniques increase the network capacity by using the resources in a more effective way than in single user transmissions. Unlike orthogonal division multiple access (OFDMA) used in the DL in Long Term Evolution (LTE) networks, NOMA takes advantages of the channel rate quantization, allowing an UE to access a portion of the power resources allocated to another UE, and that do not help improving the data rate of the latter. The principle behind NOMA consists on pairing UEs with large channel gain difference; this difference is then translated into multiplexing gain [11]. In NOMA UEs are multiplexed in the same time/frequency resources; this is possible by implementing superposition transmission schemes and adaptive power allocation in the transmitter. The power ratio assigned to an allocated UE will depend on its channel conditions; the lower the channel gain, the higher the power ratio. Fig. 1 shows a resource allocation comparison between OMA and NOMA for two UEs. In the receiver, interference cancellation (IC) techniques are used.
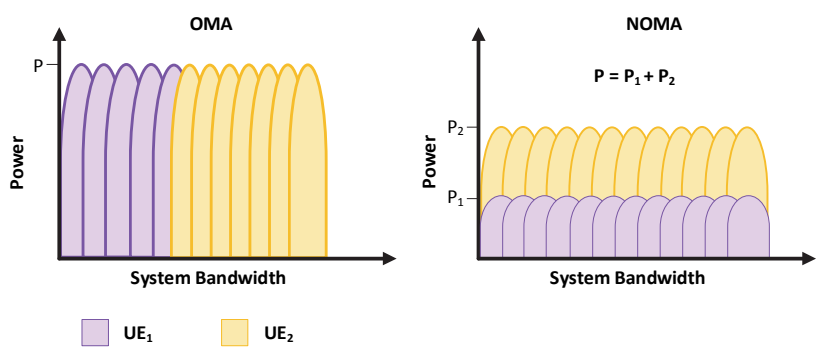

Fig. 1 Resource allocation comparison for OMA and NOMA for two UEs.

\section{B. Superposition transmission}

Superposition transmission is a physical layer technique that allows the transmitter to simultaneously send independent signals to different UEs. The number of UEs selected to be allocated in the same time/frequency resources can vary depending on the system configuration. However, the more users are paired together, the more interference there will be in the received signal, resulting in a higher BLER and hence in an increased number of retransmissions. After implementing superposition transmission for two UEs, the transmitted signal would be as follows:

$$
x=\sqrt{P_{1}} x_{1}+\sqrt{P_{2}} x_{2}
$$

where $x_{i}$ and $P_{i}$ are the signal from $\mathrm{UE}_{\mathrm{i}}$ and its power ratio, respectively; the maximum transmission power $P_{T X_{\max }}=P_{1}+P_{2}$. If no adaptive power is implemented in the transmitter, $P_{i}=P_{T X \max }$.

\section{Receiver Schemes}

The joint transmissions in NOMA cause a large amount of interference in the received signal. To mitigate the interference effect, IC methods can be applied. The received signal by $\mathrm{UE}_{\mathrm{i}}$ will be of the form:

$$
y_{i}=h_{i} x+w_{i}
$$

where $x$ is the transmitted superposed signal, $h_{i}$ represents the complex channel coefficient between the $\mathrm{UE}_{\mathrm{i}}$ and the base station (BS), and $w_{i}$ represents the Gaussian noise plus interference received by $\mathrm{UE}_{\mathrm{i}}$. The optimal order for decoding the received signal in NOMA, is in the order of increasing channel gain normalized by the noise and inter-cell interference power, $\left|h_{i}\right|^{2} / N_{0, i}$ [2]. Assuming that $\mathrm{UE}_{1}$ has better channel conditions than $\mathrm{UE}_{2},\left|h_{1}\right|^{2} / N_{0,1}>\left|h_{2}\right|^{2} / N_{0,2}$, then the signal from the $\mathrm{UE}_{2}$ would be the first in the decoding order. Therefore, $\mathrm{UE}_{2}$ can decode its message from the superpositioned signal with a linear receiver, treating the signal from $\mathrm{UE}_{1}$ in $y_{2}$ as additional interference. For $\mathrm{UE}_{1}$, the decoding process consists on first decoding and reconstructing the $\mathrm{UE}_{2}$ signal, and subtracting it from $y_{1}$.

\section{SYSTEM MODEL}

In the model developed, a single cell was considered with LTE as the wireless technology; only the DL transmission was analyzed. The number of UEs was limited to 50, and they were randomly located following a normal distribution with mean $\mu=25$, and standard deviation $\sigma=10$. For simplicity reasons, no mobility was considered; the reason for this is that since the channel can considerably vary when using mmWave, an approach as the one proposed with these frequencies would be limited to stationary or semi-stationary environments. The size of the packet to be transmitted to the scheduled UEs was chosen randomly, from 16 to 97880 bits. If the TBS assigned to a UE was not enough to send the whole packet, a total of bits equal to the TBS were sent and the rest of the bits were buffered. For the PRBs allocation, the UEs were divided into four groups according to the modulation order and the PRBs assigned to each group were proportional to the number of UEs; a minimum of two PRBs was set.

The MCS assignment and resources allocation in the model is done in two steps. In the first step a preliminary assignment is done and only the channel quality indicator (CQI) reported by each UE is used as a reference; the UEs map the wideband SINR calculated through simulations into a CQI value. The minimum SINR for each CQI was estimated in the model under constrains of a 10\% BLER and assuming an OMA transmission (without considering co-cahnnel interference). In the second step the system evaluates which UEs, if any, are candidates for NOMA. To determine this, a pairing method based on MCS adjustment and extra Tx power allocation is implemented. Such pairing guarantees that the throughput of each UE using NOMA either remains the same as in OMA or increases.

\section{A. Proposed pairing algortihm with MCS adjustment and extra Tx power allocation}

The purpose of implementing a MCS adjustment when pairing the UEs in NOMA, is to compensate for the higher SINR needed, in comparison to OMA, to successfully decode the received superposed signal [5]. In the proposed pairing algorithm all possible pairs are tested, starting with those with the highest channel gain difference, e.g., $\mathrm{UE}_{1}$ with 256QAM and $\mathrm{UE}_{2}$ with QPSK. The condition to determine whether two UEs can be paired or not, is that the throughput of each UE must not be degraded. To do this the transport block size (TBS) 
must remain the same or be bigger than with OMA. If the MCS is adjusted to a more robust value, the only way to guarantee the same TBS is to assign more physical resource blocks (PRBs). When in NOMA, more PRBs can be assigned to a UE by pairing it with another UE. However, as the extra SINR needed is on average $12 \mathrm{~dB}$ [5], if the MCS adjustment is not enough to cover such difference while guaranteeing that the throughput will not be degraded, there is the possibility that only a few (i.e., 2 or 4) or none of the UEs can be paired. Moreover, it is important to consider that at least one of the paired UEs has to use QPSK as modulation [5]; otherwise the superposed constellation becomes too complex to decode. This only lowers the possibilities of finding suitable UEs to pair.

To overcome this limitation, extra Tx power allocation can be considered. For example, let us assume that for OMA, the $\mathrm{BS}$ is not transmitting at the maximum regulated power, because lower Tx power is enough to guarantee the desired BLER and to cover the desired area. Then, a Tx power headroom can be considered in a hybrid MA system for the subcarriers that are using NOMA, as long as the total Tx power does not exceed the regulated maximum limit. By allocating extra Tx power when needed in NOMA, it is then possible to provide the extra dBs that cannot be provided with the MCS adjustment to reach the desired SINR. Although allocating extra Tx power to some UEs could be considered going against the idea of having $5 \mathrm{G}$ networks that are more energy efficient, if such extra power does not exceed the regulations, it could help increasing the probabilities of having UEs paired which impacts directly on the total channel capacity.

For the proposed pairing method to work effectively, extra signaling information needs to be shared between the BS and the UEs. Since the BS does not know the exact value of the SINR that the UE experiences, the latter needs to inform the BS if the MCS adjustment is enough or if extra Tx power needs to be sent to cover the extra dBs needed in the SINR. Table 1 shows the grouping method used to classify the UEs during the pairing process; this method aims at giving priority to the UEs with the larger difference in channel gain. A total of six iterations are run to test all the possible pairs as long as the modulations of the UEs are different. In Fig. 2 the logical process of the pairing algorithm is shown.

\section{B. User bit rate}

The rate of each UE depends on the MA method that was used for their scheduling. Assuming that a UE occupies $\beta$ of the total bandwidth, $B$, during a subframe, then the bit rate for that UE with OMA can be calculated as:

$$
R_{\text {OMA }}=B * \beta * \log _{2}(1+\operatorname{SINR})
$$

With NOMA, the UE rate depends as well on the power allocated, $\alpha$, and can be calculated as [6]:

$$
R_{\text {NOMA }}=B * \beta * \log _{2}(1+(\alpha * \operatorname{SINR}))
$$

Table 1 Modulation of the UEs $\in$ Group $X$, with $X=1,2$ for each iteration $i$ of the proposed pairing method

\begin{tabular}{|c|c|c|c|c|c|c|}
\hline$i$ & 1 & 2 & 3 & 4 & 5 & 6 \\
\hline Group 1 & 256QAM & 256QAM & 256QAM & 64QAM & 64QAM & 16QAM \\
\hline Group 2 & QPSK & 16QAM & 64QAM & QPSK & 16QAM & QPSK \\
\hline
\end{tabular}

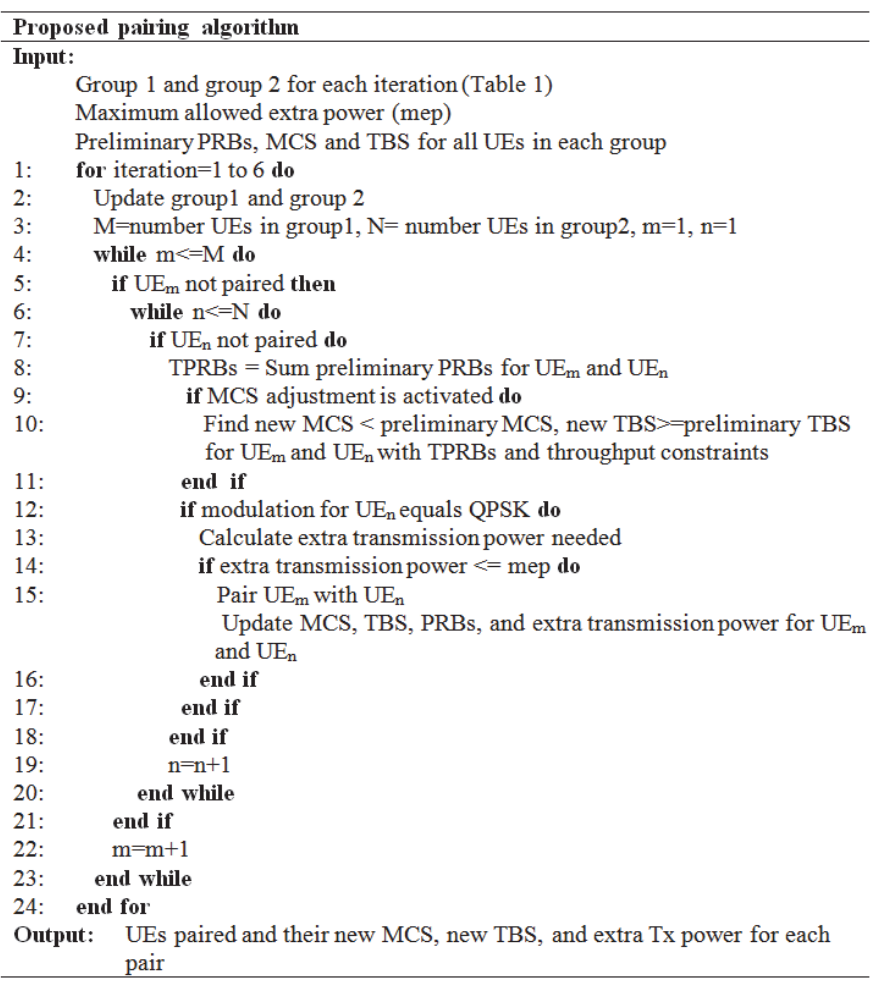

Fig. 2 Proposed pairing algorithm for NOMA based on MCS adjustment and extra $\mathrm{T} x$ power allocation

\section{Performance Evaluation}

Table 2 shows the six study cases considered for the evaluation of the hybrid MA system, where the percentage of maximum extra Tx power is based on the Tx power for OMA. In Table 3, the extra Tx power for case 6 is based only the value reported by the UE to reach the extra $12 \mathrm{~dB}$ needed in the SINR. The propagation parameters used for the simulations are shown in Table 3; $\mathrm{UE}_{1}$ is assumed to be the UE with highest channel gain. The performance evaluation was based on four system aspects: the UE's bit rate, the overall system capacity, the required extra Tx power, and the BLER. For all the results shown in this paper, an OMA only system was used as benchmark. The results are shown in Fig3 - Fig 6 and they were averaged over different runs of the model, with 100 subframes being transmitted in each run.

If we focus on the UEs bit rate, we can see from Fig. 3 that with the case 6 some of the UEs can experience up to a 30 -fold increase in their bit rate, with $90 \%$ of the UEs experiencing increases up to 9.4-fold. The reason for the high increase in this case is that there is no limit in the extra Tx power that can be allocated to the NOMA UEs, therefore two UEs which require a large amount of extra power can still be paired. Hence, their bit rate will significantly increase since NOMA is more effective as the difference in the channel gains is larger. Case 5 was the one that offered the lowest performance, with no UEs paired. The reason for this is that the maximum $10 \%$ extra power for this case was not enough to make up for the extra $\mathrm{dBs}$ needed in the SINR. Cases 2 and 3 showed a similar performance, and the same trend was shown for cases 1 and 4 . Table 4 summarizes the results shown in Fig. 3 for the $90^{\text {th }}$, $80^{\text {th }}$, and $50^{\text {th }}$ percentile. 
Table 2 Study cases

\begin{tabular}{|c|c|c|c|c|c|c|}
\hline Case & 1 & 2 & 3 & 4 & 5 & 6 \\
\hline MCS adjusment & Yes & Yes & Yes & Yes & Yes & No \\
\hline $\begin{array}{c}\text { Maximum extra } \\
\text { TX power }\end{array}$ & $0 \%$ & $75 \%$ & $50 \%$ & $25 \%$ & $10 \%$ & Unlimited \\
\hline
\end{tabular}

Table 3 Simulation parameters [12]

\begin{tabular}{|c|c|}
\hline Carrier Frequency & $73 \mathrm{GHz}$ \\
\hline Channel Bandwidth & $800 \mathrm{MHz}$ \\
\hline Coding /Decoding & Turbo coding \\
\hline Modulation Scheme & QPSK, 16QAM, 64QAM, 256QAM \\
\hline Maximum DL Tx Power & $30 \mathrm{dBm}$ \\
\hline DL Tx Power & $15 \mathrm{dBm}$ \\
\hline \multirow{2}{*}{ Power allocation factor per UE } & OMA: 1 \\
\hline & NOMA: 0.25 for UE1 and 0.75 for UE2 \\
\hline Waveform & OFDM \\
\hline Transmission mode & SISO \\
\hline TX Antenna Gain & $37 \mathrm{dBi}$ \\
\hline Path Loss Model & $\mathrm{P}_{\mathrm{L}}=69.8 \mathrm{~dB}+\mathrm{A} \log (\mathrm{d})+\mathrm{x}_{\sigma}{ }^{1}$ \\
\hline Channel estimation & MMSE \\
\hline RX Antenna Gain & $0 \mathrm{dBi}$ \\
\hline Noise figure & $6 \mathrm{~dB}$ \\
\hline \multirow{2}{*}{ Receiver type } & OMA: LMMSE \\
\hline & NOMA: SLIC for UE1 and LMMSE-IRC for UE2 \\
\hline
\end{tabular}

$1 \mathrm{x}_{\sigma}$ represents the shadowing factor and it is a radom Gaussian variable with mean zero and standard deviation $\sigma=5.2 \mathrm{~dB}$ for LOS and $\sigma=57.6 \mathrm{~dB}$ for NLOS. For LOS the constant $\mathrm{A}=20$ and for NLOS $\mathrm{A}=33$ [12]

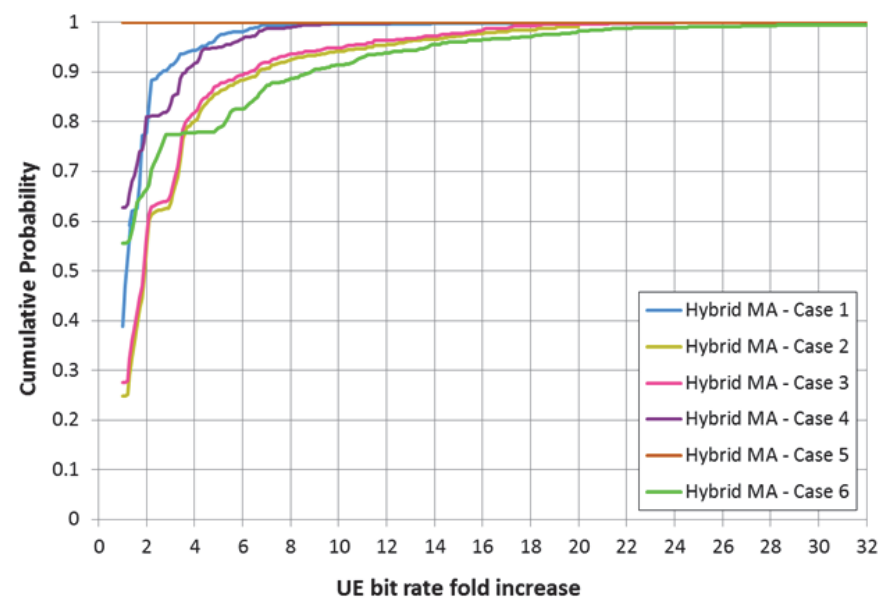

Fig. 3 Cumulative probability for the UEs bit rate fold increase for a hybrid MA system, using an OMA only system as benchmark

Table 4 UEs bit rate fold increase for the $90^{\text {th }}, \mathbf{8 0}^{\text {th }}$ and $50^{\text {th }}$ percentile for a hybrid MA system, using an OMA only system as benchmark

\begin{tabular}{|c|c|c|c|c|c|c|c|}
\cline { 2 - 8 } \multicolumn{1}{c|}{} & Case & 1 & 2 & 3 & 4 & 5 & 6 \\
\hline \multirow{3}{*}{ Percentile } & 90 th & 2.7 & 6.7 & 6.3 & 3.8 & 1 & 9.4 \\
\cline { 2 - 8 } & 80 th & 2.1 & 4 & 3.7 & 2 & 1 & 5.3 \\
\cline { 2 - 8 } & 50 th & 1.1 & 1.9 & 1.9 & 1 & 1 & 1 \\
\hline
\end{tabular}

If we now analyze the overall system capacity, we can see from Fig. 4 that case 2 is the one that offers the best performance with a 1.78-fold increase, corresponding to a channel capacity of approximately $6.7 \mathrm{Gbps}$. If we also look at Fig. 5 which shows the UEs pairing probability, we can see that case 2 is the one with the highest pairing probability, along with case 1 , with 0.4 . For case 2 this is because when combining the MCS adjustment with a high percentage of extra Tx power (e.g., 75\%) is more likely to reach the average extra dBs needed in the SINR values, which also impacts on the UEs bit rate (equations 3 and 4). For case 1, although it also showed the highest pairing probability, as it does not implement extra Tx power allocation, the UEs bit rate is lower than in case 2, as confirmed in Fig. 4, with a 1.12-fold increase. Case 3, with $50 \%$ maximum extra Tx Power, offered a pairing probability similar to cases 1 and 2, with 0.46 for a 1.72 fold increase in the system. For case 5 there was no gain in the system capacity, since no UEs met the requirements to be paired.

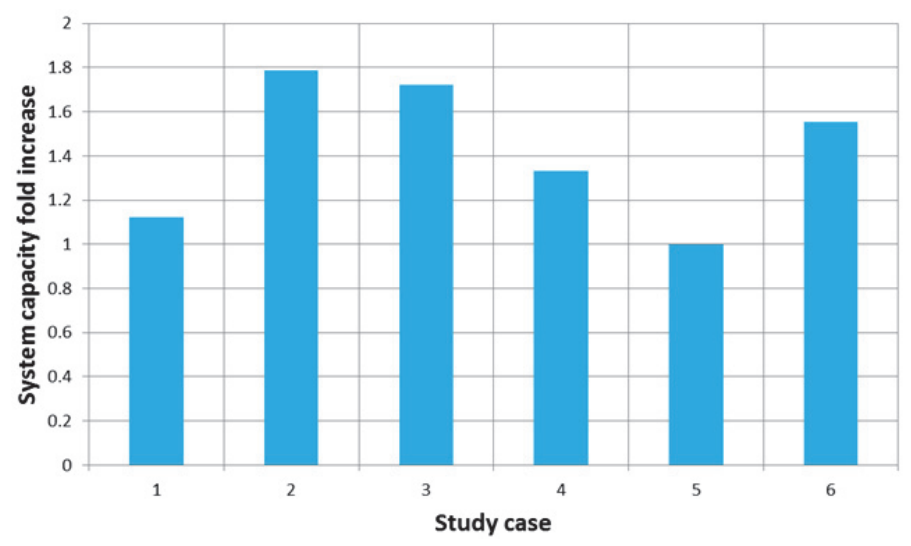

Fig. 4 System capacity fold increase for a hybrid MA system, using an OMA only system as benchmark

The performance for cases 4 and 6 was very similar with 1.33 and 1.55 -fold increase in the system capacity, and pairing probabilities of 0.24 and 0.28 , respectively. The reason for this behavior is that, although for case 6 the UEs bit rate was superior than for case 4, there is no MCS adjustment in case 6. As stated earlier, at least one of the paired UEs in NOMA has to have QPSK as modulation. When MCS adjustment is implemented, some of the UEs with higher order modulations can be assigned a QPSK modulation, which increases the probability of having candidates to be paired for NOMA.

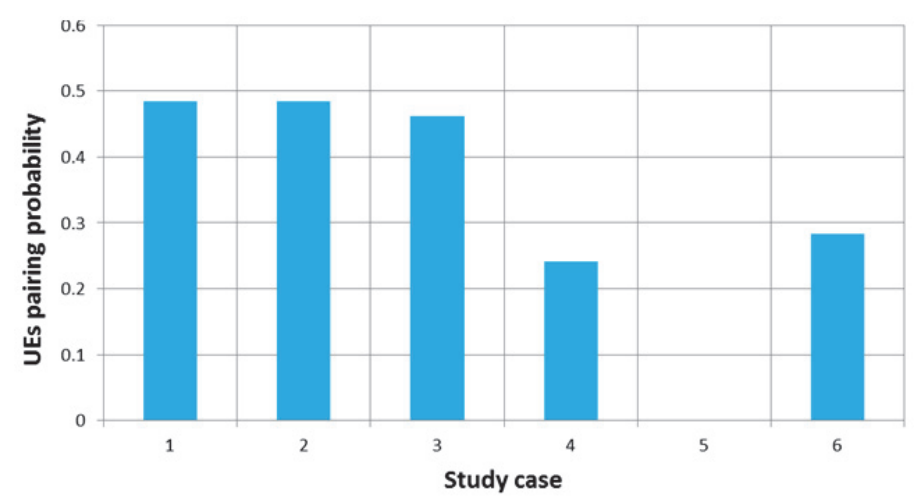

Fig. 5 UEs pairing probability for a hybrid MA system

From a power consumption point of view, the results are shown in Fig. 6. For case 1 there is no extra Tx power allocated, while for case 6 there is no limit in the amount of extra power that can be assigned for the NOMA transmissions. We can see that case 6 requires an average of $73 \%$ extra power. For case 5 a higher power allocation should be considered if 
the main concern relies on the overall system capacity. A higher power allocation along with the CQI adjustment leads to more NOMA UEs and a higher system capacity. Case 4 requires on average $14 \%$ of extra Tx power, $11 \%$ less that the maximum allowable. This is an indication that this amount of maximum extra power could offer a good tradeoff between power consumption and system capacity. Cases 2 and 3, require on average less power than the maximum allowable, with $19 \%$ and $18 \%$, respectively. These results confirm that no more than $14-19 \%$ of extra Tx power would be necessary to expect a significant performance improvement. Finally, from a BLER perspective, all the cases except case 1 offered a BLER below $10 \%$, which is usually the maximum allowable. For case 1 , the BLER could reach values up to $30 \%$; this is because since there is no extra power allocation for this case, some of the paired UEs require extra $\mathrm{dBs}$ to reach the SINR values needed to successfully decode the received superposed signal.

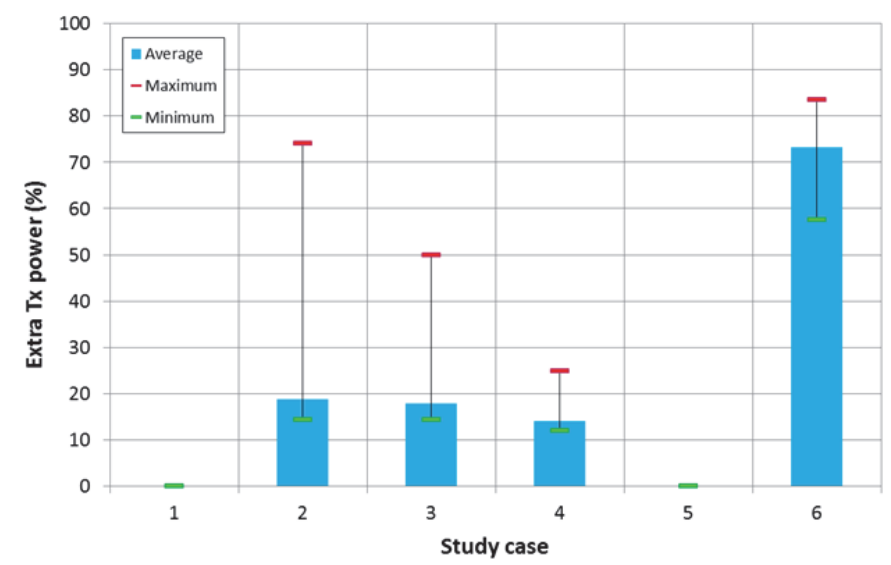

Fig. 6 Extra Tx power for the NOMA UEs in a hybrid MA system

From the results analysis we can conclude that best tradeoffs between the UEs bit rates, overall system capacity, power consumption, and BLER, are achieved when a hybrid MA system is implemented along with MCS adjustment and extra Tx power allocation for the NOMA transmissions. In the case of applying MCS adjustment but not extra Tx power allocation, the BLER values might be higher than the maximum desired, which eventually affects the QoS since the number of HARQ retransmissions will increase. If on the contrary, no MCS adjustment in performed, and all the needed extra Tx power is allocated, there would be an improvement in the system performance but not a significant one if compared with other cases, at the expenses of using on average $73 \%$ extra Tx power.

We therefore suggest an implementation of a hybrid MA system with a pairing algorithm based on MCS adjustment and an allowed extra Tx power allocation between $14 \%$ and $19 \%$ (e.g., cases 2, 3 and 4), since with this configuration the UEs bit rates average fold increase could be between 1.75-3.31, the overall system capacity could also increase between 1.33 and 1.78-fold, corresponding to approximately 5.1-6.7 Gbps, and the BLER will remain below $10 \%$.

With this proposed method a significant gain can be achieved in the overall system capacity when a Tx power headroom is available. Moreover, the only additional signaling needed, in comparison with any NOMA implementation, is to verify whether the MCS adjustment was enough for the UE to achieve the desired SINR with NOMA or if extra Tx power is needed. Hence, the impact of this method in the signaling overhead is expected to be low.

\section{CONCluSions}

In this paper we presented the performance evaluation of a hybrid MA system, combining OMA and NOMA, combined with a proposed pairing algorithm based on MCS adjustment and extra Tx power allocation techniques. The purpose of using these techniques is to aid the UEs reaching the extra dBs needed in the SINR when NOMA is implemented instead of OMA. Moreover, we used mmWave for the signal propagation to further increase the system capacity. We evaluated six cases for the hybrid MA system: one with only MCS adjustment and no extra Tx power allocation; one with unlimited extra Tx power allocation and no MCS adjustment, and four combining both proposed techniques for different percentages of maximum extra Tx power. The results show that implementing the proposed hybrid MA system jointly with the MCS and power adjustment offers the best tradeoff in terms of UEs bit rate, overall system capacity, power consumption and BLER. Moreover, we show that allowing an extra Tx power allocation between $14 \%$ and $19 \%$ can offer the best performance, with a system capacity gain up to 1.78 -fold for an approximate of 6.7 Gbps, an average UE bit rate increase up to 3.31-fold, and a BLER below $10 \%$.

\section{REFERENCES}

[1] ITU, "IMT Vision - Framework and overall objectives of the future development of IMT for 2020 and beyond, M Series, Recommendation ITU-R M.2083-0 (09/2015)," vol. 0, 2015.

[2] Y. Saito, Y. Kishiyama, A. Benjebbour, T. Nakamura, A. Li, and K. Higuchi, "Non-orthogonal multiple access (NOMA) for cellular future radio access," IEEE Veh. Technol. Conf., pp. 0-4, 2013.

[3] “3GPP TR 36.859: Study on Downlink Multiuser SuperposItion Transmission (MUST) for LTE (Release 13)," no. V13.0.0. 2016.

[4] Z. Ding, Y. Liu, J. Choi, Q. Sun, M. Elkashlan, C.-L. I, and H. V. Poor, "Application of Non-orthogonal Multiple Access in LTE and 5G Networks," IEEE Commun. Mag., vol. 55, no. 2, pp. 185-191, 2017.

[5] A. S. Marcano and H. L. Christiansen, "Performance of NonOrthogonal Multiple Acess in mmWave wireless communications for $5 \mathrm{G}$ networks," in IEEE ICNC, 2017.

[6] Y. Yuan, Z. Yuan, G. Yu, C. Hwang, P. Liao, A. Li, and K. Takeda, "Non-Orthogonal Transmission Technology in LTE Evolution," IEEE Commun. Mag., no. July, pp. 68-74, 2016.

[7] W. Shin, M. Vaezi, B. Lee, D. J. Love, J. Lee, and H. V. Poor, "NonOrthogonal Multiple Access in Multi-Cell Networks: Theory, Performance, and Practical Challenges," IEEE Commun. Mag., 2016.

[8] M. Vaezi and H. V. Poor, "Simplified Han-Kobayashi region for onesided and mixed Gaussian interference channels," in 2016 IEEE International Conference on Communications (ICC), 2016.

[9] Y. Sun, D. W. K. Ng, Z. Ding, and R. Schober, "Optimal Joint Power and Subcarrier Allocation for Full-Duplex Multicarrier NonOrthogonal Multiple Access Systems," IEEE Trans. Commun., 2017.

[10] Z. Wei, D. W. K. Ng, and J. Yuan, "Power-Efficient Resource Allocation for MC-NOMA with Statistical Channel State Information," in IEEE GLOBECOM, 2016.

[11] NTT Docomo, "5G Radio Access: Requirements, Concept and Technologies,” no. July, pp. 1-13, 2014.

[12] T. S. Rappaport, R. W. Heath Jr., C. R. Daniels, and N. J. Murdock, Millimeter Wave - Wireless Communications. Prentice Hall, 2014. 